\title{
Glicerina bruta no suplemento e seus efeitos nas características da carcaça e nos componentes do peso vivo de cordeiros lactentes
}

\author{
[Crude glycerin in supplement and its effects on carcass characteristics and live \\ weight components in suckling lambs] \\ A.C.R.S. Pellegrin ${ }^{1}$, C.C. Pires ${ }^{2}$, R.O. Mello ${ }^{2}$, L. Müller ${ }^{2}$, S. Carvalho ${ }^{2}$, J.F. Lopes ${ }^{1}$ \\ ${ }^{1}$ Aluna de pós-graduação - Universidade Federal de Santa Maria - Santa Maria, RS \\ ${ }^{2}$ Universidade Federal de Santa Maria - Santa Maria, RS
}

\begin{abstract}
RESUMO
Objetivou-se avaliar o efeito da porcentagem de glicerina bruta em suplementos sobre as características da carcaça e os componentes do peso vivo de cordeiros lactentes mantidos em pasto de azevém. Foram utilizados 32 cordeiros lactentes, distribuídos nos tratamentos: 0, 10, 20 e 30\% de glicerina bruta na matéria seca, em substituição ao milho, no suplemento isoproteico (18\% de PB na matéria seca) fornecido diariamente em quantidade equivalente a $2 \%$ do peso corporal. As porcentagens de glicerina bruta tiveram efeito linear decrescente sobre peso de carcaça quente, peso de carcaça fria, rendimento de carcaça quente e rendimento de carcaça fria, enquanto as perdas pelo jejum aumentaram. O peso da paleta e o peso do costilhar diminuíram, enquanto a proporção de perna aumentou com o incremento de glicerina bruta no suplemento. Na composição tecidual da paleta, observou-se que a gordura subcutânea, a gordura intermuscular e a gordura total da paleta decresceram linearmente segundo as porcentagens de glicerina bruta. Sobre os componentes do peso vivo, observaram-se diferenças nas proporções de pele, rúmen, omaso, intestino grosso, que aumentaram, enquanto a de gordura renal diminuiu linearmente. Conclui-se que o uso de glicerina bruta em até $30 \%$ em suplemento fornecido em comedouro privativo para cordeiros lactentes mantidos em pasto de azevém interfere nas características da carcaça e nos componentes corporais.
\end{abstract}

Palavras-chave: biodiesel, componentes não carcaça, glicerol, rendimento

\begin{abstract}
The objective was to evaluate the effect of crude glycerin levels in supplement on carcass characteristics and live weight components of suckling lambs maintained on ryegrass pasture. Thirty two suckling lambs were used and were distributed among the treatments: 0, 10, 20 and 30\% crude glycerin, in replacement of corn, in the isoproteic supplement (18\% CP) provided daily in amounts equivalent to $2 \%$ of body weight. The levels of crude glycerin had negative linear effects on hot and cold carcass weight, hot and cold carcass yield but an increasing effect was observed on gastrointestinal content and fasting losses. The shoulder and rib weights decreased, while the leg proportion increased with the elevation of crude glycerin use. In the tissue composition of the shoulder, it was observed that the subcutaneous fat, intermuscular fat and total fat decreased linearly according to the levels of crude glycerin. Regarding body weight components, differences were observed in the proportions of skin, rumen, omasum, large intestine, which increased, while the kidney fat decreases linearly. The use of crude glycerin in up to $30 \%$ in the supplement provided in creep feeding for suckling lambs kept in ryegrass pasture influences carcass characteristics and corporal components.
\end{abstract}

Keywords: biodiesel, glycerol, non-carcass components, yield

Recebido em 15 de agosto de 2012

Aceito em 2 de fevereiro de 2013

E-mail: carolsanquetta@hotmail.com 


\section{INTRODUÇÃO}

Nos sistemas de produção de carne, as características quali-quantitativas da carcaça são importantes, e estão diretamente relacionadas ao produto final, sendo úteis principalmente como parâmetros para que o consumidor possa identificar a qualidade do produto (Sousa et al., 2009).

Na produção de cordeiros, é necessário que haja um bom manejo alimentar que permita rápida terminação desses animais para a obtenção de carcaças com características adequadas ao consumo (Frescura et al., 2005). Contudo, os diversos sistemas de produção de carne e a qualidade da dieta fornecida têm influenciado não apenas nas características da carcaça dos cordeiros, mas também nos componentes não integrantes da carcaça ou componentes de peso vivo (Neres et al., 2001; Yamamoto et al., 2004).

O efeito da nutrição sobre a composição física da carcaça tem sido muito estudado e tem-se verificado que animais com melhor regime alimentar apresentam carcaças de melhor qualidade, visto que tiveram maior desenvolvimento muscular, boa deposição de gordura e menor proporção de ossos (Fernandes et al., 2010). Entretanto, melhorar a nutrição para os cordeiros, por meio da utilização de suplementos convencionais, pode elevar os custos de produção (Carvalho et al., 2006), o que motiva o uso de alimentos alternativos na nutrição desses animais.

Sendo assim, com o aumento na produção de biodiesel, a disponibilidade da glicerina bruta, seu maior subproduto, aumenta de forma gradativa, e possivelmente haverá redução no valor pago por ela, tornando-a um produto acessível para uso na nutrição animal.

O interesse do uso da glicerina bruta na nutrição animal é pelo fato de ela ter o potencial para substituir parcialmente os ingredientes à base de amido na dieta, como o milho, pois o glicerol, que compõe, em média, $85 \%$ da matéria seca da glicerina bruta, é convertido em propionato no rúmen e atua como um precursor para a síntese hepática de glicose. Porém, seus efeitos sobre as características da carcaça dos animais ainda não foram bem definidos (Gunn et al., 2010).

A presente pesquisa foi conduzida com $o$ objetivo de avaliar o efeito de porcentagens de inclusão da glicerina bruta em suplementos sobre as características da carcaça e os componentes do peso vivo de cordeiros lactentes mantidos em pasto de azevém.

\section{MATERIAL E MÉTODOS}

Esta pesquisa foi realizada no Laboratório de Ovinocultura do Departamento de Zootecnia da Universidade Federal de Santa Maria, Santa Maria - RS e foi aprovada pela Comissão de Ética no Uso de Animais da mesma instituição (Protocolo 006/2011).

Avaliaram-se quatro tratamentos, correspondentes às porcentagens de glicerina bruta, em substituição ao milho, no suplemento fornecido em comedouros privativos para cordeiros, sendo estes: suplemento com 0, 10, 20 e 30\% de glicerina bruta na matéria seca (Tab. 1).

Tabela 1. Proporções dos ingredientes e composição química dos suplementos experimentais fornecidos a cordeiros lactentes mantidos a pasto

\begin{tabular}{lcccc}
\hline \multirow{2}{*}{ Item } & \multicolumn{4}{c}{ Percentuais de glicerina bruta (\%) } \\
\cline { 2 - 5 } Ingrediente & 0 & 10 & 20 & 30 \\
\hline Glicerina bruta & 0 & $\%$ de matéria seca & 30 \\
Grão de milho moído & 73,33 & 61,49 & 20 & 39,66 \\
Farelo de soja & 25,37 & 27,21 & 29,04 & 30,88 \\
Calcário calcítico & 1,30 & 1,30 & 1,30 & 1,30 \\
\hline Composição química & \multicolumn{5}{c}{$\%$ de matéria seca } \\
\hline Matéria seca & 89,05 & 88,99 & 88,93 & 88,86 \\
Proteína bruta & 18,00 & 18,00 & 18,00 & 18,00 \\
Extrato etéreo & 9,11 & 8,10 & 7,08 & 6,07 \\
Cálcio & 0,53 & 0,54 & 0,54 & 0,54 \\
Fósforo & 0,33 & 0,32 & 0,32 & 0,31 \\
Energia líquida (Mcal/kg de matéria seca) & 2,10 & 2,10 & 2,09 & 2,08 \\
\hline
\end{tabular}


As características físico-químicas da glicerina bruta utilizada foram: $84,8 \%$ de glicerol, $89 \%$ de matéria seca, $5,1 \%$ de cinzas, $2,1 \%$ de lipídeos totais, $0,06 \%$ de proteína bruta, $0,0045 \%$ de cálcio, $0,055 \%$ de fósforo, $0,025 \%$ de potássio, 2,29\% de sódio e 0,0019 de magnésio, $0 \%$ de álcool, pH de 5,67, densidade de 1,248g/mL e $2,20 \mathrm{Mcal}$ de energia líquida/kg, matéria seca calculada de acordo com Mach et al. (2009). Esta era proveniente de usina produtora de biodiesel que utilizava a soja como matéria-prima.

Como animais experimentais, foram utilizados 32 cordeiros lactentes (machos e fêmeas), mantidos com suas mães até o abate, tendo peso médio inicial de $12,32 \pm 1,59 \mathrm{~kg}$, sendo estes distribuídos igualmente de acordo com sexo, tipo de parto e peso entre os tratamentos. Adotou-se o delineamento experimental em blocos ao acaso, com quatro tratamentos e quatro repetições (animais) por bloco, totalizando oito repetições por tratamento.

Os animais foram mantidos em pasto de azevém anual (Lolium multiflorum Lam.), em sistema de lotação contínua com carga animal variável (Mott e Lucas, 1952), sendo estes mantidos continuamente na área experimental, enquanto o número de animais reguladores variou para ajuste da carga animal, o qual foi realizado a cada 14 dias, visando manter a massa de forragem em $1.200 \mathrm{~kg}$ de MS/ha.

O suplemento isoproteico $(18 \%$ de $\mathrm{PB})$ foi fornecido uma vez ao dia, no comedouro privativo, em quantidade equivalente a $2 \%$ do peso corporal (PC) para cada cordeiro.

Os animais foram deixados em restrição alimentar de sólidos quando atingiram peso para que o abate fosse realizado, sendo o peso de abate preestabelecido de $28 \mathrm{~kg}$. Foram determinados o peso vivo (PV: peso vivo sem jejum) e o peso vivo ao abate (PVA) com os animais em jejum prévio de sólidos por 14 horas. As perdas pelo jejum (PPJ) foram obtidas por meio de cálculo correspondente à diferença entre $\mathrm{o}$ peso vivo de fazenda e o peso vivo ao abate $\mathrm{e}$ expressas em \% do PC.

Em seguida, os animais foram insensibilizados e, então, abatidos. $\mathrm{O}$ sangue de cada animal foi recolhido em recipiente apropriado $\mathrm{e}$ pesado. Após esfola e evisceração, pesou-se separadamente cada componente corporal, e suas proporções foram calculadas em relação ao peso de corpo vazio. O conteúdo gastrointestinal, a bile e a urina foram descartados para obtenção do peso corporal vazio (PCV): $\mathrm{PCV}=\mathrm{PVA}-$ (conteúdo gastrointestinal + bile + urina). Posteriormente, realizou-se a pesagem da carcaça para obtenção do peso de carcaça quente (PCQ). Pela relação entre o PVA e o PCQ, obteve-se o rendimento de carcaça quente $(\mathrm{RQ}=(\mathrm{PCQ} / \mathrm{PVA})$ $\times 100)$. Em seguida, as carcaças foram levadas à refrigeração em câmara frigorífica a $2^{\circ} \mathrm{C}$ por 24 horas.

Transcorrido o período de resfriamento, as carcaças foram novamente pesadas para obtenção do peso de carcaça fria $(\mathrm{PCF})$, do rendimento de carcaça fria $(\mathrm{RF}=(\mathrm{PCF} / \mathrm{PVA})$ $\times 100)$ e do índice de quebra ao resfriamento $(\mathrm{IQR}=100-((\mathrm{PCF} / \mathrm{PCQ}) \times 100)$. Determinaram-se, ainda, como características subjetivas, o estado de engorduramento da carcaça, que expressa a quantidade e a distribuição harmônica da gordura na carcaça $(1,0=$ excessivamente magra a $5,0=$ excessivamente gorda), e a conformação da caraça $(1=$ muito pobre a $5=$ excelente), que indica o desenvolvimento das massas musculares (Osório et al., 1998).

Posteriormente, as carcaças foram seccionadas longitudinalmente ao meio, obtendo-se, assim, duas meias carcaças. Em seguida, procedeu-se às medições relativas às características quantitativas e qualitativas da carcaça dos animais (Osório et al., 1998). Na metade esquerda, mensurou-se o comprimento de carcaça, e o índice de compacidade da carcaça (ICC) foi determinado por meio do PCF em função do comprimento da carcaça $(\mathrm{CC}),(\mathrm{ICC}=\mathrm{PCF} / \mathrm{CC})$, sendo expresso $\mathrm{em} \mathrm{kg} / \mathrm{cm}$.

A área de olho-de-lombo (AOL) foi obtida pela exposição do músculo Longissimus dorsi após um corte transversal na carcaça entre a $12^{\mathrm{a}}$ e $13^{\mathrm{a}}$ costelas, traçando o contorno do músculo em papel vegetal. A área foi, então, calculada com auxílio do programa computacional AutoCAD (AutoCAD release 14.0, versão R14.0.0, copyright 1982 - 1997, Autodesk, Inc.), com leitura em mesa digitalizadora. No músculo Longissimus dorsi, mediante a observação visual, foi determinada a gordura de marmoreio (gordura intramuscular ou gordura de infiltração) 
em uma escala de 1 a 5 , em que 1,0 = inexistente e $5,0=$ excessivo.

Em seguida, procedeu-se à separação regional da meia carcaça direita em quatro cortes: perna, paleta, costilhar e pescoço, sendo o corte das paletas de acordo com Colomer-Rocher et al. (1988) e os demais de acordo com Osório et al. (1998). Cada corte foi pesado individualmente, e posteriormente suas proporções foram calculadas em relação ao peso da carcaça fria. Os dois músculos Longissimus dorsi foram retirados inteiros de cada meia carcaça e, então, pesados, obtendo-se, assim, o peso total de lombo.

As paletas foram separadas, identificadas e congeladas para posterior determinação da composição tecidual delas. Depois de descongelada, cada paleta foi pesada e, em seguida, procedeu-se à separação física com o auxílio do bisturi e pinça em: gordura subcutânea, gordura intermuscular e gordura préescapular, e a soma destas foi considerada a gordura total, osso, músculo e outros tecidos (vasos, nervos, gânglios linfáticos, tendões, aponeuroses e fáscias), de acordo com ColomerRocher et al. (1988). Cada um dos componentes teciduais que compunha as paletas foi pesado, e sua proporção calculada em relação ao corte.

Todas as variáveis foram corrigidas para o peso vivo ao abate. Os dados de cada variável foram submetidos à análise da variância a 0,05 de significância, e os resultados significativos foram submetidos à análise de regressão, em que se testaram os efeitos linear, quadrático e cúbico. $\mathrm{O}$ sexo e o tipo de parto dos animais foram incluídos no modelo estatístico como efeito fixo. Todas as análises foram realizadas por intermédio do pacote estatístico SAS (Statistical Analysis System, versão 9.2)..

\section{RESULTADOS E DISCUSSÃO}

Não houve efeito $(\mathrm{P}>0,05)$ das porcentagens de glicerina bruta sobre peso vivo sem jejum e peso vivo ao abate, sendo isto esperado devido ao fato de o peso de abate ser preestabelecido ( $28 \mathrm{~kg}$ ). Por outro lado, as perdas pelo jejum aumentaram, enquanto o peso de carcaça quente, o peso de carcaça fria, o rendimento de carcaça quente e o rendimento de carcaça fria decresceram linearmente em razão do aumento da porcentagem de glicerina bruta (Tab. 2).

Tabela 2. Características da carcaça de cordeiros lactentes mantidos a pasto que receberam suplementos com glicerina bruta

\begin{tabular}{|c|c|c|c|c|c|c|c|}
\hline \multirow[t]{2}{*}{ Variável } & \multicolumn{4}{|c|}{$\begin{array}{c}\text { Percentuais de glicerina } \\
\text { Bruta }(\%)\end{array}$} & \multicolumn{3}{|c|}{$P$} \\
\hline & 0 & 10 & 20 & 30 & Linear & Quadrático & Cúbico \\
\hline Dias na pesquisa (dias) & 48 & 53 & 56 & 61 & 0,1374 & 0,9292 & 0,9343 \\
\hline Peso vivo sem jejum (kg) & 28,79 & 29,72 & 29,66 & 29,30 & 0,3431 & 0,618 & 0,5072 \\
\hline Peso vivo ao abate $(\mathrm{kg})$ & 27,97 & 28,44 & 28,35 & 27,99 & 0,9704 & 0,3863 & 0,8387 \\
\hline Perdas pelo jejum $(\%)^{1}$ & 2,85 & 4,30 & 4,42 & 4,47 & 0,0197 & 0,1457 & 0,3381 \\
\hline Conteúdo GI+B+U, $\mathrm{kg}^{2}$ & 2,93 & 2,69 & 3,12 & 3,24 & 0,0295 & 0,1685 & 0,0743 \\
\hline Peso de carcaça quente $(\mathrm{kg})^{3}$ & 14,66 & 14,36 & 14,08 & 13,73 & 0,0003 & 0,8832 & 0,9346 \\
\hline Peso de carcaça fria $(\mathrm{kg})^{4}$ & 14,21 & 13,89 & 13,59 & 13,23 & $<0,0001$ & 0,9025 & 0,8957 \\
\hline Rendimento de carcaça quente $(\%)^{5}$ & 51,52 & 51,07 & 50,02 & 48,90 & 0,0013 & 0,5560 & 0,8371 \\
\hline Rendimento de carcaça fria $(\%)^{6}$ & 49,94 & 49,37 & 48,29 & 47,11 & 0,0003 & 0,5661 & 0,8686 \\
\hline IQR $(\%)$ & 3,16 & 3,29 & 3,41 & 3,64 & 0,2254 & 0,8606 & 0,9203 \\
\hline $\mathrm{ICC}(\mathrm{kg} / \mathrm{cm})$ & 0,26 & 0,26 & 0,25 & 0,25 & 0,1215 & 0,2726 & 0,7699 \\
\hline Conformação & 2,85 & 3,01 & 2,84 & 2,85 & 0,7754 & 0,5801 & 0,4240 \\
\hline Engorduramento & 3,18 & 3,25 & 2,94 & 2,93 & 0,1130 & 0,7875 & 0,3027 \\
\hline EGS (mm) & 1,75 & 1,99 & 1,44 & 1,41 & 0,1226 & 0,4994 & 0,1868 \\
\hline Marmoreio & 2,76 & 2,54 & 2,24 & 2,64 & 0,4778 & 0,3034 & 0,4057 \\
\hline $\mathrm{AOL}\left(\mathrm{cm}^{2}\right)$ & 13,36 & 12,98 & 12,75 & 12,73 & 0,4498 & 0,7519 & 0,2640 \\
\hline Peso total de lombo $(\mathrm{kg})$ & 0,850 & 0,848 & 0,836 & 0,854 & 0,6765 & 0,6744 & 0,9627 \\
\hline
\end{tabular}

IQR: índice de quebra ao resfriamento, ICC: índice de compacidade de carcaça, EGS: espessura de gordura subcutânea, AOL: área de olho-de-lombo.

${ }^{1} \hat{\mathrm{Y}}=3,33+0,05 \mathrm{~GB}\left(\mathrm{R}^{2}=0,20\right),{ }^{2} \hat{\mathrm{Y}}=2,78+0,01 \mathrm{~GB}\left(\mathrm{R}^{2}=0,15\right),{ }^{3} \hat{\mathrm{Y}}=14,67-0,03 \mathrm{~GB}\left(\mathrm{R}^{2}=0,41\right),{ }^{4} \hat{\mathrm{Y}}=14,21-0,03 \mathrm{~GB}$ $\left(\mathrm{R}^{2}=0,51\right),{ }^{5} \hat{\mathrm{Y}}=51,71-0,09 \mathrm{~GB}\left(\mathrm{R}^{2}=0,52\right),{ }^{6} \hat{\mathrm{Y}}=50,11-0,09 \mathrm{~GB}\left(\mathrm{R}^{2}=0,59\right)$. 
Os resultados podem ser justificados por diferenças no conteúdo gastrointestinal dos animais desta pesquisa. Para Martinez et al. (2001), este fator e o grau de acabamento das carcaças são os fatores que mais influenciam no peso e no rendimento delas.

Além disso, o conteúdo gastrointestinal é maior quanto menor for a digestibilidade dos alimentos ingeridos pelos animais (Brochier e Carvalho, 2008), indicando que pode haver alterações na digestibilidade da dieta pelo uso da glicerina bruta (Lage et al., 2010; D'aurea et al., 2010), o que justifica também as diferenças encontradas para a variável relacionada às perdas pelo jejum observadas na Tab. 3, que se elevou à medida que se aumentou a glicerina bruta no suplemento.

As diferentes proporções nos componentes do peso vivo (rúmen, omaso e intestino grosso) também justificam os resultados encontrados e serão discutidas posteriormente.

Diferenças nas características da carcaça dos cordeiros foram encontradas por Musselman et al. (2010), tendo os cordeiros que receberam as menores inclusões de glicerina bruta (0 e 15\%) os maiores rendimentos de carcaça, o que corrobora esta pesquisa. Vale salientar que diferenças no rendimento de carcaça são importantes do ponto de vista mercadológico e que menores rendimentos acabam prejudicando o resultado final obtido pelo sistema produtivo (Carvalho et al., 2007).

Os resultados alcançados para o índice de quebra ao resfriamento não diferiram entre as porcentagens de glicerina bruta $(\mathrm{P}>0,05)$ e obtiveram como média 3,37\%, sendo considerados dentro do aceitável para Almeida Jr. et al. (2004), pois, de acordo com esses autores, as perdas pelo resfriamento devem estar entre 3,0 e 4,0\%. A semelhança estatística encontrada pode estar associada também aos dados obtidos para estado de engorduramento e espessura de gordura subcutânea $(\mathrm{P}>0,05)$, que mantiveram os índices de quebra ao resfriamento próximos. Para Silva Sobrinho et al. (2005), as perdas pelo resfriamento são maiores em carcaças com menor gordura de cobertura.
O índice de compacidade da carcaça também não foi influenciado $(\mathrm{P}>0,05)$ pelas porcentagens de glicerina bruta, com média de $0,25 \mathrm{~kg} / \mathrm{cm}$. Macedo et al. (2008) consideram que não se deve esperar diferenças significativas para esse índice, principalmente quando os animais são do mesmo grupo genético ou são abatidos com mesmo peso, como é o caso desta pesquisa.

Não houve efeito $(\mathrm{P}>0,05)$ dos percentuais de glicerina bruta sobre as características da carcaça relacionadas à conformação, estado de engorduramento, espessura de gordura subcutânea, marmoreio, área de olho-de-lombo e peso total de lombo dos cordeiros (Tab. 2). A não ocorrência de diferenças significativas para essas características pode ser atribuída ao fato de os animais terem sido abatidos com mesmo peso e idade bastante próxima (Macedo et al., 2008), pois não houve diferenças significativas no número de dias em que os animais levaram para serem abatidos aos $28 \mathrm{~kg}$.

Ainda, ressalta-se que a glicerina bruta é rapidamente convertida em propionato no rúmen, o que ocasiona diminuição na relação acetato:propionato, sendo plausível considerar que a glicerina bruta pode causar efeitos sobre depósitos de gordura corporal (Gunn et al., 2010). Porém, mesmo assim, não houve efeito da inclusão dela sobre o estado de engorduramento e a espessura de gordura subcutânea, que obtiveram média de 3,08 grau e $1,65 \mathrm{~mm}$, respectivamente.

Houve efeito dos percentuais de glicerina bruta sobre o peso de paleta $(\mathrm{P}<0,01)$, o peso de costilhar $(\mathrm{P}<0,001)$ e a proporção de perna $(\mathrm{P}<0,05)$ (Tab. 3). O peso de paleta e o de costilhar diminuíram linearmente com o aumento de glicerina bruta. Essa diferença no peso do costilhar pode estar associada à diminuição na deposição de gordura nele, pois a deposição de gordura na carcaça influencia no peso do costilhar. Ribeiro et al. (2009) e Frescura et al. (2005) ressaltam que o aumento de proporção de costilhar não é desejado, pois o valor comercial é inferior ao dos outros cortes, sendo a perna a parte mais nobre na carcaça ovina. 
Tabela 3. Pesos e proporções dos cortes comerciais da carcaça de cordeiros lactentes mantidos a pasto que receberam suplementos com glicerina bruta

\begin{tabular}{lccccccc}
\hline \multirow{2}{*}{\multicolumn{1}{c}{ Variável }} & \multicolumn{9}{c}{ Percentuais de glicerina bruta } \\
& 0 & 10 & 20 & 30 & Linear & Quadrático & Cúbico \\
\cline { 2 - 8 } & 0,497 & 0,526 & 0,520 & 0,483 & 0,6280 & 0,1474 & 0,9801 \\
\hline Pescoço $(\mathrm{kg})$ & 1,466 & 1,449 & 1,434 & 1,370 & 0,0094 & 0,3401 & 0,6607 \\
Paleta $(\mathrm{kg})$ & 2,635 & 2,615 & 2,491 & 2,454 & 0,0017 & 0,8509 & 0,3363 \\
Costilhar $(\mathrm{kg})^{2}$ & 2,298 & 2,383 & 2,332 & 2,255 & 0,4339 & 0,1133 & 0,6262 \\
Perna (kg) & 7,25 & 7,40 & 7,63 & 7,41 & 0,5147 & 0,4314 & 0,6278 \\
Pescoço (\%) & 21,15 & 20,73 & 21,52 & 20,83 & 0,8874 & 0,6707 & 0,1056 \\
Paleta (\%) & 38,11 & 37,46 & 36,89 & 37,27 & 0,2299 & 0,3687 & 0,7396 \\
Costilhar $(\%)$ & 33,50 & 33,67 & 34,33 & 34,64 & 0,0179 & 0,8785 & 0,6230 \\
Perna $(\%)^{3}$ & ${ }^{1} \hat{\mathrm{Y}}=1,47-0,003 \mathrm{~GB}\left(\mathrm{R}^{2}=0,30\right),{ }^{2} \hat{\mathrm{Y}}=2,65-0,0067 \mathrm{~GB}\left(\mathrm{R}^{2}=0,54\right),{ }^{3} \hat{\mathrm{Y}}=33,43+0,041 \mathrm{~GB}\left(\mathrm{R}^{2}=0,38\right)$. &
\end{tabular}

Além disso, o peso da carcaça dos cordeiros também deve ser considerado, pois na medida em que este diminui, reduzem-se em valores absolutos os pesos de osso, músculo e gordura e, consequentemente, o peso das regiões da carcaça ou cortes comerciais (Osório et al., 2002). Ribeiro et al. (2009) ao compararem sistemas de terminação de cordeiros abatidos aos $32 \mathrm{~kg}$, obtiveram resultados para pesos de pescoço $(0,652 \mathrm{~kg})$, paleta $(1,502 \mathrm{~kg})$, costilhar $(2,776 \mathrm{~kg}) \mathrm{e}$ perna $(2,388 \mathrm{~kg})$ superiores aos encontrados nesta pesquisa e os mesmos autores também afirmam que o peso dos cortes está diretamente relacionado ao peso da carcaça. Desta maneira, a diferença significativa observada no peso da carcaça entre os tratamentos pode justificar a diminuição no peso de paleta e costilhar da carcaça (Tab. 3).

Já em relação a proporções dos cortes, observase que a proporção de perna aumentou linearmente com o acréscimo de glicerina bruta, mesmo sem o peso de perna sofrer influência dos tratamentos, a proporção deste corte aumentou de maneira compensatória, provavelmente pela diminuição nos pesos de paleta e costilhar e devido ao menor peso de carcaça fria.

Devido ao oneroso trabalho de dissecar totalmente uma meia carcaça, a determinação da composição tecidual foi realizada pela avaliação da paleta, devido seus altos coeficientes de correlação com a composição da carcaça e pela facilidade de sua obtenção (Cañeque e Sañudo, 2005).
Houve efeito $(\mathrm{P}<0,05)$ dos porcentuais de glicerina bruta sobre a composição tecidual da paleta dos cordeiros (Tab. 4).

A gordura subcutânea expressa tanto em $\mathrm{kg}$ como em proporção $(\mathrm{P}<0,001)$, a gordura intermuscular em $\mathrm{kg}(\mathrm{P}<0,05)$ e a gordura total da paleta, em $\mathrm{kg}(\mathrm{P}<0,01)$ e em proporção $(\mathrm{P}<0,05)$ decresceram linearmente com $\mathrm{O}$ aumento das porcentagens de glicerina bruta.

Um dos motivos que explicaria o ocorrido seria devido à substituição do milho pela glicerina bruta de acordo com o aumento da inclusão desta, pelo fato do milho apresentar teores mais elevados de lipídeos $(10,97 \%)$ que a glicerina bruta utilizada $(2,10 \%)$ e devido á alterações na relação ruminal de acetato:propionato já citadas.

Além do menor peso de carcaça observado entre os tratamentos, este menor peso de paleta pode ter sido em função de uma menor deposição de gordura na paleta dos cordeiros, pois tanto peso de paleta como deposição de gordura foram decrescendo com o aumento no uso de glicerina bruta (Tab. 4).

A relação músculo:osso da paleta não sofreu influência dos tratamentos $(\mathrm{P}>0,05)$, enquanto que a relação músculo:gordura da paleta dos cordeiros aumentou com o acréscimo de glicerina bruta nos suplementos $(\mathrm{P}<0,05)$ (Tab. 4), devido a menor deposição de gordura na paleta desses animais dos animais explicada anteriormente. 
Glicerina bruta...

Tabela 4. Peso, composição tecidual, relação músculo:osso e músculo:gordura da paleta de cordeiros lactentes mantidos a pasto que receberam suplementos com glicerina bruta

\begin{tabular}{|c|c|c|c|c|c|c|c|}
\hline \multirow[t]{2}{*}{ Variável } & \multicolumn{4}{|c|}{$\begin{array}{l}\text { Percentuais de glicerina bruta } \\
\qquad(\%)\end{array}$} & \multicolumn{3}{|c|}{$P$} \\
\hline & 0 & 10 & 20 & 30 & Linear & Quadrático & Cúbico \\
\hline Paleta $(\mathrm{kg})^{1}$ & 1,464 & 1,457 & 1,424 & 1,367 & 0,0073 & 0,3196 & 0,9904 \\
\hline Músculo (kg) & 0,789 & 0,810 & 0,775 & 0,759 & 0,1709 & 0,0958 & 0,3256 \\
\hline Músculo (\%) & 53,96 & 55,51 & 54,38 & 55,70 & 0,1947 & 0,8676 & 0,1034 \\
\hline Gordura subcutânea $(\mathrm{kg})^{2}$ & 0,111 & 0,106 & 0,096 & 0,083 & 0,0008 & 0,4983 & 0,8963 \\
\hline Gordura subcutânea $(\%)^{3}$ & 7,69 & 7,22 & 6,67 & 6,14 & 0,0085 & 0,9401 & 0,9592 \\
\hline Gordura intermuscular $(\mathrm{kg})^{4}$ & 0,119 & 0,116 & 0,112 & 0,100 & 0,0354 & 0,5050 & 0,8150 \\
\hline Gordura intermuscular (\%) & 8,24 & 7,91 & 7,80 & 7,38 & 0,1869 & 0,9220 & 0,7887 \\
\hline Gordura pré-escapular (kg) & 0,053 & 0,048 & 0,046 & 0,042 & 0,0542 & 0,9931 & 0,7845 \\
\hline Gordura pré-escapular (\%) & 3,65 & 3,28 & 3,23 & 3,10 & 0,1725 & 0,6690 & 0,7671 \\
\hline Gordura total $(\mathrm{kg})^{5}$ & 0,283 & 0,271 & 0,254 & 0,224 & 0,0029 & 0,5425 & 0,8909 \\
\hline Gordura total $(\%)^{6}$ & 19,59 & 18,41 & 17,71 & 16,62 & 0,0302 & 0,9625 & 0,8490 \\
\hline Osso $(\mathrm{kg})$ & 0,261 & 0,252 & 0,265 & 0,249 & 0,6254 & 0,3727 & 0,3143 \\
\hline Osso $(\%)$ & 17,75 & 17,33 & 18,61 & 18,20 & 0,2817 & 0,9891 & 0,1703 \\
\hline Outros (kg) & 0,079 & 0,082 & 0,085 & 0,083 & 0,5003 & 0,6567 & 0,8641 \\
\hline Outros $(\%)$ & 5,38 & 5,56 & 5,91 & 6,13 & 0,0956 & 0,9525 & 0,8420 \\
\hline Relação músculo:osso & 3,06 & 3,23 & 2,94 & 3,07 & 0,5627 & 0,8665 & 0,2020 \\
\hline Relação músculo:gordura ${ }^{7}$ & 2,83 & 3,06 & 3,15 & 3,41 & 0,0275 & 0,9367 & 0,7000 \\
\hline
\end{tabular}

${ }^{\mathrm{T}} \hat{\mathrm{Y}}=1,47-0,003 \mathrm{~GB}\left(\mathrm{R}^{2}=0,54\right),{ }^{2} \hat{\mathrm{Y}}=0,11-0,0009 \mathrm{~GB}\left(\mathrm{R}^{2}=0,61\right),{ }^{3} \hat{\mathrm{Y}}=7,71-0,05 \mathrm{~GB}\left(\mathrm{R}^{2}=0,54\right),{ }^{4} \hat{\mathrm{Y}}=0,12-0,0006 \mathrm{~GB}$ $\left(\mathrm{R}^{2}=0,65\right),{ }^{5} \hat{\mathrm{Y}}=0,28-0,0019 \mathrm{~GB}\left(\mathrm{R}^{2}=0,70\right),{ }^{6} \hat{\mathrm{Y}}=19,52-0,10 \mathrm{~GB}\left(\mathrm{R}^{2}=0,66\right),{ }^{7} \hat{\mathrm{Y}}=2,84+0,018 \mathrm{~GB}\left(\mathrm{R}^{2}=0,61\right)$.

O aumento nas porcentagens de glicerina bruta influenciaram as proporções de pele, rúmen $(\mathrm{P}<0,01)$; omaso e intestino grosso $(\mathrm{P}<0,001)$, que aumentaram, enquanto que a de gordura renal diminui linearmente $(\mathrm{P}<0,01)$ (Tab. 5). Apesar de não ter sido verificado diferença para o número de dias até o abate dos animais, a diferença numérica existente (13 dias entre 0 e $30 \%$ ) pode ter influenciado os resultados encontrados em relação aos componentes do peso vivo, pois para Huidobro e Villapadierna (1992) a pele, intestino grosso e os estômagos possuem desenvolvimentos tardios, sendo maiores proporções destes verificados em animais mais velhos. Dados estes que também complementam os dados significativos encontrados relacionados aos pesos e rendimentos de carcaça e ás perdas pelo jejum devido ao aumento dos componentes do peso vivo.

Além disso, o aumento da concentração de glicerol nas dietas é um fator que pode ter influenciado negativamente a digestibilidade da FDN pela redução da atividade celulolítica das bactérias no rúmen, ou seja, a glicerina bruta por não possuir parede celular, apresenta maior velocidade de degradação que outras fontes energéticas provenientes de grãos. Tal fato que pode ter diminuído a atividade celulolítica e, consequentemente aumentado a atividade amilolítica para obtenção de energia, o que causa redução na digestibilidade da FDN (Lage et al., 2010; D'Aurea et al., 2010). Esta menor digestibilidade pode ter afetado o tempo de permanência dos alimentos no trato digestório e o desenvolvimento do mesmo, pois para Jenkins (1993), a alimentação durante o período de crescimento do animal altera a digestibilidade da mesma, podendo influenciar no desenvolvimento dos órgãos.

Há diminuição no peso e na proporção de gordura renal $(\mathrm{P}<0,01)$ em função das porcentagens de glicerina bruta (Tab. 5), o que demonstra o efeito da glicerina bruta sobre os depósitos de gordura. Além disso, segundo Kempster (1981) a deposição de gordura renal é tardia quando comparado com acréscimo de gordura da carcaça, sendo esta então uma das últimas a ser depositada. 
Tabela 5. Proporções (\% do peso de corpo vazio) dos componentes do peso vivo de cordeiros lactentes mantidos a pasto que receberam suplementos com glicerina bruta

\begin{tabular}{|c|c|c|c|c|c|c|c|}
\hline \multirow[t]{2}{*}{ Variável } & \multicolumn{4}{|c|}{$\begin{array}{l}\text { Percentuais de glicerina bruta } \\
(\%)\end{array}$} & \multicolumn{3}{|c|}{$P$} \\
\hline & 0 & 10 & 20 & 30 & Linear & Quadrático & Cúbico \\
\hline Cabeça & 4,12 & 4,13 & 4,18 & 4,18 & 0,5292 & 0,9349 & 0,8017 \\
\hline Língua & 0,28 & 0,29 & 0,29 & 0,28 & 0,9559 & 0,5423 & 0,9144 \\
\hline Pulmão+Traqueia & 1,58 & 1,64 & 1,58 & 1,78 & 0,6672 & 0,1031 & 0,2226 \\
\hline Diafragma & 0,50 & 0,51 & 0,52 & 0,53 & 0,1967 & 0,9702 & 0,9869 \\
\hline Timo & 0,56 & 0,56 & 0,56 & 0,53 & 0,7852 & 0,8362 & 0,9783 \\
\hline Esôfago & 0,14 & 0,13 & 0,13 & 0,13 & 0,6335 & 0,9442 & 0,8778 \\
\hline Rúmen ${ }^{1}$ & 1,44 & 1,49 & 1,56 & 1,78 & 0,0044 & 0,2972 & 0,7479 \\
\hline Retículo & 0,29 & 0,31 & 0,28 & 0,31 & 0,8502 & 0,0738 & 0,1481 \\
\hline Omaso $^{2}$ & 0,14 & 0,15 & 0,15 & 0,21 & 0,0003 & 0,7736 & 0,1929 \\
\hline Abomaso & 0,57 & 0,61 & 0,66 & 0,61 & 0,3577 & 0,3267 & 0,6242 \\
\hline Intestino delgado & 3,52 & 3,54 & 3,79 & 3,73 & 0,2056 & 0,7880 & 0,4255 \\
\hline Intestino grosso $^{3}$ & 1,09 & 1,15 & 1,24 & 1,30 & 0,0001 & 0,9199 & 0,7908 \\
\hline Fígado & 2,11 & 2,07 & 2,06 & 2,13 & 0,8446 & 0,4354 & 0,9086 \\
\hline Vesícula biliar & 0,02 & 0,02 & 0,02 & 0,02 & - & - & - \\
\hline Pâncreas & 0,16 & 0,15 & 0,14 & 0,14 & 0,0968 & 0,3168 & 0,6989 \\
\hline Baço & 0,26 & 0,20 & 0,23 & 0,22 & 0,3163 & 0,2497 & 0,2465 \\
\hline Coração & 0,55 & 0,52 & 0,59 & 0,52 & 0,8879 & 0,5004 & 0,1076 \\
\hline Rins & 0,41 & 0,38 & 0,40 & 0,39 & 0,6109 & 0,6934 & 0,2870 \\
\hline Bexiga & 0,10 & 0,11 & 0,07 & 0,08 & 0,1683 & 0,6373 & 0,1212 \\
\hline Gordura renal $^{4}$ & 0,57 & 0,56 & 0,38 & 0,36 & 0,0099 & 0,9541 & 0,2912 \\
\hline Gordura pericárdica & 0,21 & 0,19 & 0,25 & 0,21 & 0,6626 & 0,6702 & 0,2523 \\
\hline Gordura interna & 1,81 & 1,96 & 1,83 & 2,10 & 0,2483 & 0,5992 & 0,3754 \\
\hline Aparelho reprodutor & 0,56 & 0,56 & 0,50 & 0,49 & 0,0628 & 0,8534 & 0,1794 \\
\hline Patas & 2,62 & 2,86 & 2,98 & 2,91 & 0,2386 & 0,3953 & 0,9585 \\
\hline Pelego $^{5}$ & 10,23 & 10,61 & 10,67 & 11,46 & 0,0063 & 0,4739 & 0,4274 \\
\hline Sangue & 5,32 & 5,34 & 5,57 & 5,49 & 0,3513 & 0,7874 & 0,5148 \\
\hline
\end{tabular}

${ }^{1} \hat{\mathrm{Y}}=1,41+0,01 \mathrm{~GB}\left(\mathrm{R}^{2}=0,25\right),{ }^{2} \hat{\mathrm{Y}}=0,13+0,002 \mathrm{~GB}\left(\mathrm{R}^{2}=0,35\right),{ }^{3} \hat{\mathrm{Y}}=1,09+0,007 \mathrm{~GB}\left(\mathrm{R}^{2}=0,39\right),{ }^{4} \hat{\mathrm{Y}}=0,59-0,008 \mathrm{~GB}$ $\left(\mathrm{R}^{2}=0,21\right),{ }^{5} \hat{\mathrm{Y}}=10,18+0,04 \mathrm{~GB}\left(\mathrm{R}^{2}=0,23\right)$.

\section{CONCLUSÕES}

Porcentagens de glicerina bruta em até $30 \%$, em substituição ao milho, no suplemento fornecido em comedouro privativo para cordeiros lactentes mantidos em pasto de azevém, interferiram nos pesos e nos rendimentos de carcaça e nos componentes corporais dos cordeiros. O fornecimento de glicerina bruta afetou a deposição de gordura na paleta e de gordura renal dos cordeiros.

\section{AGRADECIMENTOS}

Os autores agradecem à empresa Granol, pelo fornecimento da glicerina bruta, aos técnicos Giuliano Fernandes Zagonel, Elisa Maria Suchek e Lidiane Toporowicz e à DBIO-TECPAR, pela realização das análises da glicerina bruta.

\section{REFERÊNCIAS}

ALMEIDA JUNIOR, G.A.; COSTA, C.; MONTEIRO, A.L.G. et al. Desempenho, características de carcaça e resultado econômico de cordeiros criados em creep feeding com silagem de grãos úmidos de milho. Rev. Bras. Zootec., v.33, p.1048-1059, 2004.

BROCHIER, M.A.; CARVALHO, S. Peso e rendimento dos componentes do peso vivo de cordeiros terminados em confinamento com dietas contendo proporções crescentes de resíduo úmido de cervejaria. Arq. Bras. Med. Vet. Zootec., v.60, p.1213-1218, 2008.

CAÑEQUE, V.; SAÑUDO, C. Estandarización de las metodologías para evaluar la calidad del producto (animal vivo, canal, carne y grasa) en los ruminantes. Madri: INIA, 2005. 448p. (Serie Ganadera, 3). 
CARVALHO, S.; BROCHIER, M.; PIVATO, J. et al. Ganho de peso, características da carcaça e componentes não-carcaça de cordeiros da raça Texel terminados em diferentes sistemas alimentares. Cienc. Rural, v.37, p.821-827, 2007.

CARVALHO, S.; VERGUEIRO, A.; KIELING, R. et al. Desempenho e características da carcaça de cordeiros mantidos em pastagem de tifton-85 e suplementados com diferentes níveis de concentrado. Rev. Bras. Agrocienc., v.12, p.357361, 2006.

COLOMER-ROCHER, F.; DELFA, R.; SIERRA, I. Método normalizado para el estudio de los caracteres cuantitativos y cualitativos de las canales, según los sistemas de produccíon. In: Método normalizado para el estudio de los caracteres cuantitativos y cualitativos de las canales caprinas y ovinas. Madrid: INIA, 1988. p.19-41.

D'AUREA, A.P.; EZEQUIEL, J.M.B.; FAVARO, V.R. et al. Digestibilidade de dietas contendo glicerina, resíduo da produção do biodiesel. In: REUNIÃO ANUAL DA SOCIEDADE BRASILEIRA DE ZOOTECNIA, 47., 2010. Anais... Salvador, Sociedade Brasileira de Zootecnia. [2010]. (CD-ROM).

FERNANDES, M.A.M.; MONTEIRO, A.L.G.; POLI, C.H.E.C. et al. Composição tecidual da carcaça e perfil de ácidos graxos da carne de cordeiros terminados a pasto ou em confinamento. Rev. Bras. Zootec., v.39, p.16001609, 2010.

FRESCURA, R.B.M.; PIRES, C.C.; SILVA, J.H.S et al. Avaliação das proporções dos cortes da carcaça, características da carne e avaliação dos componentes do peso vivo de cordeiros. Rev. Bras. Zootec., v.34, p.167-174, 2005.

GUNN, P.J.; NEARY, M.K.; LEMENAGER, R.P. et al. Effects of crude glycerin on performance and carcass characteristics of finishing wheter lambs. J. Anim. Sci., v.88, p.1771-1776, 2010.

HUIDOBRO, F.R., VILLAPADIERNA, A. Estudios sobre crescimento y desarrolo en corderos de raza Manchega. Madrid, 1992. 191p. Tesis (Doctoral) - Facultad de Veterinaria, Universidad Complutense, 1992.

JENKINS, T.C. Lipid metabolism in the rumen. J. Dairy Sci., v.76, p.3851-3863, 1993.
KEMPSTER, A.J. Fat partition and distribution in the carcasses of cattle, sheep and pigs: A review. Meat Sci., v.5, p.83-98, 1981.

LAGE, J.F.; PAULINO, P.V.; PEREIRA, L.G.R. et al. Glicerina bruta na dieta de cordeiros terminados em confinamento. Pesq. Agrop. Bras., v.45, p.1012-1020, 2010.

MACEDO, V.P.; SILVEIRA, A.C.; GARCIA, C.A. et al. Desempenho e características de carcaça de cordeiros alimentados em comedouros privativos recebendo rações contendo semente de girassol. Rev. Bras. Zootec., v.37, p.2041-2048, 2008.

MACH, N.; BACH, A.; DEVANT, M. Effects of crude glycerin supplementation on performance and meat quality of Holstein bulls fed highconcentrate diets. J. Anim. Sci., v.87, p.632-638, 2009.

MARTINEZ, D.E.; NUÑEZ, F.A.G.; GARCÍA, A.M. et al. Caracterización de canales de borregos alimentados con desechos de papel. Rev. Bras. Agrocienc., v.7, p.50-53, 2001.

MOTT, G.O.; LUCAS, H.L. The design, conduct, and interpretation of grazing trials in cultivated and improved pastures. In: INTERNATIONAL GRASSLAND CONGRESS, 6., State College. Proceedings... State College: Pensylvania, State College Press. 1952. p.13801385.

MUSSELMAN, A.F.; VAN EMON, M.L.; GUNN, P.J. et al. Effects of crude glycerin on feedlot performance and carcass characteristics of market lambs. In: AMERICA SOCIETY OF ANIMAL SCIENCE, 59., 2008, Laramie, WY. Proceedings... Laramie: Western Section, 2008. p.353-355.

NERES, M.A.; MONTEIRO, A.L.G.; GARCIA, C.A. et al. Forma física da ração e pesos de abate nas características da carcaça de cordeiros em creep feeding. Rev. Bras. Zootec., v.30, p.948954, 2001.

OSÓRIO, J.C.S.; OSÓRIO, M.T.M.; JARDIM, P.O.C. et al. Métodos para avaliação da produção de carne ovina: "in vivo" na carcaça e na carne. Pelotas: Ed. UFPEL, 1998. 107p.

OSÓRIO, J.C.S.; OSÓRIO, M.T.M.; OLIVEIRA, N.M. et al. Qualidade, morfologia e avaliação de carcaças. Pelotas: Universidade Federal de Pelotas, 2002. 194p. 
RIBEIRO, T.M.D.; COSTA, C.; MONTEIRO, A.L.G. et al. Componentes não constituintes da carcaça e cortes cárneos de cordeiros em diferentes sistemas de alimentação. Boletim Ind. Anim., v.66, p.11-19, 2009.

SAS INSTITUTE. SAS/STAT: user's Guide. Version 9.2. Cary: SAS Institute, 2009. 7869p.

SILVA SOBRINHO, A.G.; PURCHAS, R.W.; KADIM, I.T. et al. Musculosidade e composição da perna de ovinos de diferentes genótipos e idades de abate. Pesq. Agrop. Bras., v.40, p.1129-1134, 2005.
SOUSA, W.H.; BRITO, E.A.; MEDEIROS, A.N. et al. Características morfométricas e de carcaça de cabritos e cordeiros terminados em confinamento. Rev. Bras. Zootec., v.38, p.13401346, 2009.

YAMAMOTO, S.M.; MACEDO, F.A.F.; MEXIA, A.A. et al. Rendimentos dos cortes e não-componentes das carcaças de cordeiros terminados com dietas contendo diferentes fontes de óleo vegetal. Cienc. Rural, v.34, p.1909-1913, 2004. 